\title{
EFFICACY AND SAFETY OF TETRAHYDROCURCUMINOID IN THE TREATMENT OF ORAL LEUKOPLAKIA: A PILOT STUDY
}

\author{
YOGESH CHHAPARWAL ${ }^{1 *}$, KEERTHILATHA M PAI ${ }^{1}$, M.S. KAMATH ${ }^{2}$, SUNITHA CARNELIO ${ }^{3}$, SHUBHA CHHAPARWAL ${ }^{4}$ \\ ${ }^{1}$ Department of Oral Medicine and Radiology, Manipal College of Dental Sciences, Manipal, Karnataka, India. ${ }^{2}$ Department of Ayurveda, \\ Kasturba Medical College, Manipal, Karnataka, India. ${ }^{3}$ Department of Oral and Maxillofacial Pathology, Manipal College of Dental \\ Sciences, Manipal, Karnataka, India. ${ }^{4}$ Department of Conservative Dentistry and Endodontics, Manipal College of Dental Sciences, Manipal, \\ Manipal Academy of Higher Education, Manipal, Karnataka, India. E-mail: yogesh.omr@gmail.com
}

Received: 26 June 2018, Revised and Accepted: 27 July 2018

\section{ABSTRACT}

Objective: The objective of this study is to evaluate the efficacy and safety of tetrahydrocurcuminoid (THC) in the treatment of oral leukoplakia.

Methods: Patients with oral mucosal lesions with clinical features of leukoplakia were selected, and an incisional biopsy of the lesion was performed to confirm the diagnosis. Demographic data, habit history, and complete medical history were documented. Subjects were given $2 \%$ THC gel (Sami Labs, Bengaluru) with advice to apply the gel to the affected areas, 5 times daily for 12 weeks. The lesion was examined, and its characteristics were documented in a standard manner at baseline, $3,6,9$, and 12 weeks.

Results: Of the eight patients, 6 were males with age range from 40 to 70 years (mean age of 56 years). All the patients reported a reduction of burning sensation within 3 weeks of starting treatment and were completely asymptomatic by the end of the study. There was a decrease in the size of the lesion during the follow-up period. Reduction in thickness of the lesion was found in six of eight patients. Histological improvement a stage better was seen in three patients after completion of treatment. However, there was no histological improvement in four patients, and one patient progressed to mild dysplasia from hyperkeratosis without dysplasia.

Conclusion: THD when topically applied in gel form is remarkably effective in alleviating clinical symptoms. The remarkable histological improvement was seen in 3 of 8 patients.

Keywords: Curcumin, Leukoplakia, Efficacy.

(C) 2018 The Authors. Published by Innovare Academic Sciences Pvt Ltd. This is an open access article under the CC BY license (http://creativecommons. org/licenses/by/4. 0/) DOI: http://dx.doi.org/10.22159/ajpcr.2018.v11i12.28107

\section{INTRODUCTION}

Oral cancer is one of the ten most common cancers in the world and shows a marked geographic variation in occurrence [1]. Squamous cell carcinoma (SCC) is the most common type of oral cancer constituting about $90 \%$ of oral malignancies, in India, accounting for about 3-4\% of all cancers. OSCCs are preceded in the majority of cases by clinically visible potentially malignant oral mucosal lesions which may undergo subsequent pathological changes from hyperplasia through dysplasia to the invasive neoplasm. Potentially malignant lesions of the oral cavity include leukoplakia, proliferative verrucous leukoplakia, erythroplakia, and palatal changes in reverse smokers [2].

Oral leukoplakia has recently been redefined as "predominantly white lesion of the oral mucosa that cannot be characterized as any other definable lesion; some oral leukoplakias will transform into cancer." The term is strictly a clinical one and does not imply specific histopathological tissue alteration [3].

Various chemopreventive agents such as Vitamins A, E, and C, folates, and selenium have been tried in the treatment of oral leukoplakia. Traditional herbal agents are seen as potential chemopreventive agents owing to their medicinal properties. One such example is the Curcuma longa used in treatment due to its various pharmacological properties.

C. longa is a herb which is cultivated predominantly in tropical and subtropical regions of Southeast Asia. Turmeric is a household spice which is used in food preparations, and it also has medicinal properties [4]. In traditional Hindu literature, it was widely used in the treatment of sprains and swelling caused by injury, deworming agent, gallstones, and arthritis [5,6]. The major metabolite of $C$. longa is curcumin (diferuloylmethane) [7]. The mechanism of action of the role of curcumin as a chemopreventive agent is mainly through its antioxidant activity by increasing the levels of Vitamins $\mathrm{C}$ and $\mathrm{E}$ and preventing lipid peroxidation and DNA damage [8-10]. Cheng et al. evaluated the safety and efficacy of curcumin in 25 patients with various cases of potentially malignant disorders. The significant histological improvement was seen in seven cases of oral leukoplakia [11].

Holder et al. [12] discussed the metabolic products of curcumin and reported that one of the major by-products formed during the absorption in the intestine is tetrahydrocurcuminoid (THC) which is less polar than curcumin. Krishnakumar et al. [13] showed that THC showed a greater inhibitory effect than curcumin. With this background, an open-label, single arm, single-centered clinical trial was conducted to assess the efficacy and safety of THC in the treatment of oral leukoplakia.

\section{METHODS}

The present study was conducted in the Department of Oral Medicine and Radiology, Manipal College of Dental Sciences, Manipal, in collaboration with the Department of Ayurveda, Kasturba Hospital, Manipal. Approval to carry out the clinical trial was obtained from the Kasturba Hospital Ethical Committee (KHEC/102/2006). Subjects with clinical and histopathologic features consistent with oral leukoplakia and informed consent were included in the study. Subjects with presence of any concurrent infection, medical comorbidities, contraindication to biopsy, concurrent radiotherapy, and any medications within the past 3 months were excluded from the study. 
Patients with oral mucosal lesions consistent with clinical diagnosis of leukoplakia [14] were selected for the study, and an incisional biopsy of the lesion was performed to confirm the diagnosis. Demographic data, habit history, and complete medical history were documented in a pro forma specially designed for the study. The characteristics of the leukoplakic lesion including the site/location, size, extent, surface texture, and symptoms were noted in the pro forma after a thorough clinical examination. Subjects were given 2\% THC gel (Sami Labs, Bengaluru) with advice to apply on the affected sites in the oral cavity and keep it in the mouth for 30 min, 5 times daily for 12 weeks. They were instructed not to eat, drink, or gargle for $1 \mathrm{~h}$ after applying the medication and returned for a follow-up once in 3 weeks or earlier in the event of any adverse reaction associated with the application of the medicament. The adverse effect even with a single application was considered for the evaluation of drug safety. The lesion was examined, and its characteristics were documented in a standard manner. At the end of 12 weeks, clinical features of the lesion were documented, and repeat biopsy was performed from the same site for histopathologic evaluation.

All the analysis was performed using SPSS version 14 . A $p<0.05$ was considered statistically significant. Comparison of the mean size of the lesion was made using Wilcoxon signed-rank test.

\section{RESULTS}

A total of 16 patients who met the criteria were identified for recruitment in the study, and only ten patients consented to the study. One patient developed an adverse reaction in the course of the treatment and could not continue the therapy, while another patient could not complete post-treatment assessments. Out of the eight patients, 6 were males with age range from 40 to 70 years (mean age of 56.37 years). All the eight patients enrolled in the study consumed tobacco or tobaccorelated products, presented with burning sensation as their chief complaint at the start of the survey. Five of the eight patients had leukoplakia on the buccal mucosa, two patients on the lateral border of the tongue, and one patient had an extensive lesion involving buccal mucosa, alveolar ridge, and palate. The size of the leukoplakia ranged from as small as $1 \mathrm{~cm} \times 1 \mathrm{~cm}$ to as extensive as $7 \mathrm{~cm} \times 5 \mathrm{~cm}$. Five of eight patients had presented with homogenous white surface areas, and three patients had a speckled surface appearance that is mixed red and white areas. Pre-treatment histopathological diagnosis revealed three patients to have hyperkeratosis without dysplasia, three others to have mild dysplasia, and one each had moderate dysplasia and carcinoma in situ, respectively.

All the patients reported a reduction of burning sensation within three weeks of starting treatment and were completely asymptomatic by the end of the study. Change in surface characteristics varied among patients with a reduction in thickness of the lesion being the most consistent finding in six of eight patients, while no change in surface characteristics was observed in two patients.

Comparison of the size of the lesion in pre- and post-treatment phases was statistically significant as shown in Table 1.

Three of the eight patients histologically improved a stage better after completion of therapy. Of these three patients, one patient who was diagnosed to have carcinoma in situ was downstaged to mild dysplasia. Two patients were downstaged to hyperkeratosis from mild dysplasia. However, there was no histological improvement in four patients, and one patient progressed to mild dysplasia from hyperkeratosis without dysplasia as shown in Table 2.

Table 1: Comparison of the mean size of the lesion

\begin{tabular}{lll}
\hline Measure & Mean \pm SD $^{*}$ & p value** \\
\hline Pre-treatment & $7.88 \pm 11.31$ & 0.014 \\
Post-treatment & $5.72 \pm 11.89$ & \\
\hline
\end{tabular}

*SD: Standard deviation, ${ }^{* *}$ Wilcoxon Signed-rank test

\section{DISCUSSION}

Oral leukoplakia is the most common premalignant, potentially malignant, or precancerous lesion of the oral mucosa with a malignant transformation rate from various studies and locations ranging from $0.6 \%$ to $18 \%$ [15]. Hence, prevention at the premalignant stage is prudent. Curcumin safely exerts chemopreventive effects against multiple human cancer which is mainly attributed to its potent anti-inflammatory agent. Curcumin is considered as a potential chemopreventive agent as it is known to suppress transformation, proliferation, and metastasis of tumors $[16,17]$.

One of the major metabolites of curcumin which is formed during intestinal absorption is THC [12]. It may be involved in the physiological and pharmacological properties of curcumin. Krishnakumar et al. [13] demonstrated that THC showed a greater inhibitory effect on lipid peroxidation than curcumin. They concluded that THC might be the major metabolite which is involved in the physiological and pharmacological properties of curcumin. In our study, all the patients treated for burning sensation reported a reduction in the sensation and felt better symptomatically within three weeks of initiation of treatment. This shows that curcuminoids in gel form produced a reasonably early improvement with substantial improvement in the quality of life. All the patients were completely free from burning sensation after 12 weeks of treatment. Kuttan et al. reported similar reports in precancerous lesion [18] and Hastak et al. in oral submucous fibrosis [19].

Post-treatment histopathologic reports of biopsies showed that one patient who was diagnosed as carcinoma in situ before treatment was downstaged to mild dysplasia after treatment. Of the three patients diagnosed with mild dysplasia before treatment, one was downstaged to the absence of dysplasia. However, the other two patients remained at the same stage of mild dysplasia and did not show histologic improvement. Of the two patients who were graded before treatment as hyperkeratosis without dysplasia, one remained at the same stage, and the other progressed to mild dysplasia. One patient diagnosed with moderate dysplasia before treatment remained at the same stage and did not show histologic improvement. In a similar study of 3 months' duration, Cheng et al. [11] evaluated curcumin in patients with oral leukoplakia and found histological improvement in two of seven patients and one patient progressed to malignancy. One of the ten patients developed an adverse reaction in the course of the treatment and could not continue the therapy. This patient at the end of nine weeks reported with edema of the lips and face. The patient reported that the onset of swelling of the lips and face on the application of topical medicament was immediately withdrawn from the study group and recalled for a regular follow-up. Although this patient tolerated the drug well during the first two visits, the adverse reaction reported in his third visit could be attributed to a delayed hypersensitivity response to the drug. Kuttan et al. reported a negative reaction after application of curcumin in the form of ethanol extract as an ointment to external cancerous skin lesions [18].

\section{CONCLUSION}

Hence, we can conclude from our observations that THC when topically applied in gel form is remarkably effective in alleviating clinical symptoms. The remarkable histological improvement was seen in 3 of 8 patients, although there was no histological improvement in 4 patients and one patient with hyperkeratosis without dysplasia showed progression toward mild dysplasia. The small sample size was the major shortcoming of our study. Significant placebo-controlled trials will be necessary to evaluate as a potential therapeutic agent for oral leukoplakia. The results of our study, however, have shown that THC will safely provide symptomatic relief for patients with oral leukoplakia and may be an effective chemopreventive agent.

\section{AUTHORS' CONTRIBUTION}

Concept and collection of data - Yogesh Chhaparwal, Keerthilatha Pai, MS Kamath, and Sunitha Carnelio. Writing the article and critical 
Table 2: Patient characteristics, frequency and duration of the habit, and pre- and post-treatment histopathological diagnosis

\begin{tabular}{llllll}
\hline Age & Habit & Frequency/day & Duration in years & Histopathological diagnosis \\
\cline { 3 - 5 } & & & Pre-treatment & Post-treatment \\
\hline 65 & Smokeless & 4 & 30 & Carcinoma in situ & Mild dysplasia \\
63 & Smokeless & 3 & 3 & Mild dysplasia & Mild dysplasia \\
53 & Smoking & 35 & 30 & Mild dysplasia & Mild dysplasia \\
45 & Smokeless & 15 & 40 & Hyperkeratosis without dysplasia & Hyperkeratosis without dysplasia \\
56 & Smoking & 3 & 20 & Hyperkeratosis without dysplasia & Mild dysplasia \\
46 & Smoking & 4 & 3 & Moderate dysplasia & Moderate dysplasia \\
59 & Smoking & 8 & 25 & Hyperkeratosis without dysplasia & Hyperkeratosis without dysplasia \\
64 & Smokeless & 8 & 40 & &
\end{tabular}

review of the article - Yogesh Chhaparwal, Keerthilatha Pai, and Shubha Chhaparwal. Final approval of the article - Yogesh Chhaparwal.

\section{CONFLICTS OF INTEREST}

The authors declared no conflicts of interest.

\section{REFERENCES}

1. Bánóczy J, Gintner Z, Dombi C. Tobacco use and oral leukoplakia. J Dent Educ 2001;65:322-7.

2. Speight PM, Farthing PM, Bouquot JE. The pathology of oral cancer and pre-cancer. Curr Diag Path 1997;3:165-7.

3. Axell T, Pindborg JJ, Smith CJ, van der Waal I. An international collaborative group on oral white lesions. Oral white lesions with special reference to precancerous and tobacco-related lesions: Conclusions of an international symposium held in Uppsala, Sweden, 1994. J Oral Pathol Med 1996;25:49-54.

4. Govindarajan VS. Turmeric - chemistry, technology, and quality. Crit Rev Food Sci Nutr 1980;12:199-301.

5. Ammon HP, Wahl MA. Pharmacology of Curcuma longa. Planta Med 1991:57:1-7.

6. Sharma RA, Gescher AJ, Steward WP. Curcumin: The story so far. Eur J Cancer 2005;41:1955-68.

7. Chattopadhyay I, Biswas K, Bandyopadhyay U, Banerjee RK. Turmeric and curcumin: Biological actions and medicinal applications. Curr sci 2004:87:44-53.

8. Rai B, Kaur J, Jacobs R, Singh J. Possible action mechanism for curcumin in pre-cancerous lesions based on serum and salivary markers of oxidative stress. J Oral Sci 2010;52:251-6.

9. Mohan Reddy N, Rajashekhar Reddy N, Jamil K. Spicy anti- cancer spices: A review. Int J Pharm Pharm Sci 2015;7:1-6.

10. Thorat YS, Sarvagod AM, Kulkarni SV, Hosmani AH. Treatment of mouth ulcer by curcumin loaded thermoreversible mucoadhesive gel: A technical note. Int J Pharm Pharm Sci 2015;7:399-402.

11. Cheng AL, Hsu CH, Lin JK, Hsu MM, Ho YF, Shen TS, et al. Phase I clinical trial of curcumin, a chemopreventive agent, in patients with high-risk or pre-malignant lesions. Anticancer Res 2001;21:2895-900.

12. Holder GM, Plummer JL, Ryan AJ. The metabolism and excretion of curcumin (1,7-bis-(4-hydroxy-3-methoxyphenyl)-1,6-heptadiene-3,5dione) in the rat. Xenobiotica 1978;8:761-8.

13. Krishnakumar KL, Ukken MP, Manju R. A study on the effect of indole as a substituent on a keto-enol tautomer: A synthetic approach on $\beta$-diketone. Int J Pharm Pharm Sci 2017;9:219-24.

14. Axell T, Holmstrup P, Kramer JR, Pindborg JJ, Shear M. International seminar on oral leukoplakia and associated lesions related to tobacco habits. Community Dent Oral Epidemiol 1984;12:145-54.

15. Gupta PC, Mehta FS, Daftary DK, Pindborg JJ, Bhonsle RB, Jalnawalla PN, et al. Incidence rates of oral cancer and natural history of oral precancerous lesions in a 10-year follow-up study of indian villagers. Community Dent Oral Epidemiol 1980;8:283-333.

16. Aggarwal S, Ichikawa H, Takada Y, Sandur SK, Shishodia S, Aggarwal BB, et al. Curcumin (diferuloylmethane) down-regulates expression of cell proliferation and antiapoptotic and metastatic gene products through suppression of ikappaBalpha kinase and akt activation. Mol Pharmacol 2006;69:195-206.

17. Gupta SC, Kim JH, Prasad S, Aggarwal BB. Regulation of survival, proliferation, invasion, angiogenesis, and metastasis of tumor cells through modulation of inflammatory pathways by nutraceuticals. Cancer Metastasis Rev 2010;29:405-34.

18. Kuttan R, Bhanumathy P, Nirmala K, George MC. Potential anticancer activity of turmeric (Curcuma longa). Cancer Lett 1985;29:197-202.

19. Hastak K, Lubri N, Jakhi SD, More C, John A, Ghaisas SD, et al. Effect of turmeric oil and turmeric oleoresin on cytogenetic damage in patients suffering from oral submucous fibrosis. Cancer Lett 1997;116:265-9. 